\title{
Patient Attitudes Toward a Web-based System for Monitoring Chronic Wounds
}

\author{
HYUNGJIN MYRA KIM, Sc.D., ${ }^{1,2}$ JULIE C. LOWERY, Ph.D., ${ }^{1}$ \\ JENNIFER B. HAMILL, M.P.H., ${ }^{1}$ and EDWIN G. WILKINS, M.D., M.S. ${ }^{1,3}$
}

\begin{abstract}
This study evaluated patient attitudes toward a store-and-forward telemedicine system for monitoring chronic wounds treated topically, as well as chronic wounds that have been excised and surgically closed. The system involved collection and transmission via the Internet of digital photos and other patient and wound data by a nurse, and assessment by the telemedicine physician via the Web at a later time. For each patient, wound status was assessed inperson by a physician, as well as by the telemedicine system. Our hypotheses posited that patient attitudes toward telemedicine would improve over time as they become more accustomed to the telemedicine procedures. Sixty-nine patients from two Veterans Affairs medical centers participated, including inpatients and outpatients with pressure ulcers at stage 2, 3, or 4, surgically repaired pressure ulcers, diabetic foot ulcers, or venous stasis ulcers. A questionnaire developed for the study was administered to each participant at the first and third visits. Patients were generally comfortable with the use of a telemedicine system to assess wound status. Less than $25 \%$ of the patients felt that using telemedicine at home would be worse than visiting their physician in terms of the quality of care, even though about $85 \%$ of the patients felt that it was "important" or "very important" to be seen in person by a doctor for a pressure sore. Perhaps more importantly, less than $12 \%$ of the patients felt "somewhat or very unconfident" that their doctors would be able to know about the pressure sore by the use of this system instead of seeing the wound in person. Considerable effort was required for collecting the telemedicine data; however, a significant decrease was seen in time spent collecting these data from first visit to subsequent visits (mean difference $=\mathbf{2 5 . 2} \mathrm{min}$, $p<0.01$ ), and a significant difference in time spent was seen across different wound types. These findings suggest that the ideal system of care, as perceived by patients, might be routine monitoring using the telemedicine system, with occasional in-person visits to a physician, and that the value of the system may vary by wound type and setting.
\end{abstract}

\section{INTRODUCTION}

$\mathbf{P}$ RESSURE ULCERS are a significant and costly health problem among elderly and disabled populations. ${ }^{1,2}$ One of the contributors to the cost is the lack of access to timely, specialized care. Telemedicine refers to a wide range of clinical practices wherein provider and patient

\footnotetext{
${ }^{1}$ VA Center for Practice Management and Outcomes Research, Ann Arbor, Michigan.

${ }^{2}$ Center for Statistical Consultation and Research and ${ }^{3}$ Section of Plastic and Reconstructive Surgery, University of Michigan, Ann Arbor, Michigan.
} 
are geographically separated from each other, yet linked by telecommunications systems that involve various configurations of clinical applications, technology, human resources, and service organizations. ${ }^{3-6}$ The use of telemedicine systems for the timely monitoring of pressure ulcers and other wounds would improve access to care, thereby reducing the incidence and associated costs of severely infected wounds. The costs of transporting disabled patients to a hospital or physician's office would also be reduced. In addition, hospitalization days for patients who have surgery to repair their wounds may be reduced with telemedicine by monitoring patients at home or in an extended care facility.

The Web-based telemedicine system for wound care used in this study is a store-and-forward wound assessment system that, in addition to digital images, presents to the telemedicine physician a comprehensive set of clinical data, which meet the clinical requirements of practice guidelines set forth by the Agency for Health Care Policy and Research (now the Agency for Health Research and Quality). ${ }^{3}$ The system consists of three major data collection components: (1) digital photographs of the ulcer; (2) quantitative measurements of wound status (i.e., ulcer area and volume); and (3) other wound and patient data collected by a nurse (e.g., wound drainage, patient mobility, continence status, nutrition status, comorbidities, etc.).

While evaluating the accuracy of the telemedicine system for assessing wound status, ${ }^{4}$ we administered a questionnaire exploring patient attitudes toward the telemedicine system. In addition, because a single scale is generally more convenient than multiple items for measuring and relating attitudes to other variables such as outcomes of care, we also evaluated the scalability of the items in the questionnaire. Therefore, this paper reports our findings of patient attitudes and the development of a telemedicine attitude scale based on the questionnaire used in the study. The hypotheses posited that patients' attitudes toward telemedicine would improve over time as they became more accustomed to the telemedicine procedures. We also report results from the analysis of time spent collecting and transmitting the telemedicine data.

\section{MATERIALS AND METHODS}

\section{Study design and participants}

The study was a prospective, cohort design, where diagnostic evaluations of a wound were made both by a treating physician in person as well as by a remote physician using the telemedicine system. Two Veterans Affairs medical centers (Ann Arbor, MI; and Augusta, GA) participated in data collection. Eligible patients were hospital inpatients, outpatients, or nursing home residents with chronic stage 2, 3, or 4 pressure sores, postoperative wounds having undergone a tissue flap procedure for a grade 3 or 4 pressure ulcer, or diabetic ulcer. Patients with multiple wounds were included. Mentally incompetent patients were excluded from the study. All eligible patients had to give informed consent to participate in this study, and participants were reimbursed $\$ 10$ per data collection session.

Wound data for the telemedicine assessments were collected by the nurse coordinators on each study patient for a maximum of six visits, or until the wound was healed or the patient was discharged, whichever came first. Wounds were assessed at each visit using both methods (in-person and telemedicine). Six physicians participated in the study, and they rotated roles as the in-person and telemedicine evaluators. Thus each physician served an approximately equal amount of time in each role. The assessments, which were consistent with the Agency for Health Care Policy and Research guidelines, ${ }^{3}$ included determination of the presence of necrotic tissue, cellulites, osteomyelitis, and reduction in wound size. For patients with pressure ulcers who had undergone operative repair, an assessment of wound closure was also added. Data collection began at Augusta and Ann Arbor in March 1999 and concluded in June and August 2000, respectively. More detailed information on the study design and results of the diagnostic accuracy evaluation of the telemedicine system can be found in another publication. ${ }^{4}$

\section{Questionnaire administration}

A questionnaire was administered to each patient during his first and third visits. The 
questions were asked at visit 1 to obtain patients' attitudes toward telemedicine prior to any data collection for the telemedicine system, and again at visit 3, after they had had several encounters with data collection. Each patient completed the questionnaire unless the patient was not physically able to fill in his answers, in which case the nurse coordinator administered the questionnaire. Patient attitudes consisted of perceptions regarding the burden of the telemedicine system, confidence in the evaluation, and comfort with the lack of direct contact with a physician. These constructs of patient attitudes and satisfaction have been used in evaluations of other telemedicine applications. ${ }^{5,6}$

\section{Time data}

The nurse coordinators maintained logs of the time spent with the patient collecting the telemedicine data. They recorded the visit start time immediately after entering the patient room, and recorded the stop time when they left. Microsoft Access automatically recorded the time required to enter and download the data. In addition, the time spent by the telemedicine physicians reviewing data on the Web site was automatically recorded by a Web program.

\section{Data analysis}

Descriptive statistics for each question were calculated in the form of percentages across the possible responses. One of the main goals of data analysis was to determine if opinions changed after some experience with the data collection protocol. Since attitudinal data were collected at two different visits from the same subjects, it was desirable to use a statistical method accounting for the paired data. However, because only $64 \%$ (44/69) of patients completed the questionnaire at both visits, any comparison, regardless of whether or not the pairing was considered in the analysis, may be biased if patients who completed both forms were self-selected. Therefore, we started by comparing the demographic and clinical characteristics, as well as wound type and attitude distribution, of those patients who completed the questionnaire at both times with those who completed the questionnaire at first visit only. The baseline comparisons were mostly of categorical variables and, therefore, were made using Fisher's exact test.

To compare attitudes between the two time periods, we used several methods and looked for consistency in the results. Initially, we used Fisher's exact test on all available visit 1 and 3 data and also did Fisher's exact test using only those who completed both visits; both of these analyses assume independence between visits 1 and 3. Alternative methods included only patients who completed questionnaires at both visits. To assess if patient attitude was associated with time period while accounting for the pairing of data between visits, a test of symmetry based on exact method was used. ${ }^{7}$ To assess whether the change in attitudes between visits was in a favorable direction, we calculated change-scores for each question such that a positive change-score reflected an improvement in attitude. Responses to questions ranged from 1 to 3 (for questions with three possible responses) or from 1 to 5 (for questions with five possible responses). We compared the median change-score to zero using the Wilcoxon signed-rank test for paired data.

We also investigated the relationship among the questions, to determine whether an attitudinal scale could be developed from the questions, which could then be used as a single continuous variable for subsequent analysis. To assess the scalability of the attitudinal questions, internal consistency of the items was evaluated using Cronbach's alpha $(\alpha),{ }^{8}$ a summary scale was developed using a modified scoring system based on the evaluation of internal consistency, and the scale was used to evaluate the study's hypotheses that patients' attitudes were favorable (using a one-sample $t$ test) and that their attitudes improved over time (using the Wilcoxon signed-rank test for paired data).

Finally, to help with future resource planning, we examined the time spent to perform the various data collection and assessment tasks using descriptive statistics. These data were collected at every visit for each patient. A random-effects model ${ }^{9}$ was used to evaluate whether the time spent on data collection decreased in subsequent visits. The model evalu- 
ates the relationship between data collection time and visit number while adjusting for potential within-person correlation and for other potential confounding variables. All analyses were performed using Stata version 8.1 (Stata Corp., College Station, TX) and SAS version 8.02 (SAS Institute Inc., Cary, NC).

\section{RESULTS}

\section{Sample description}

Seventy patients were recruited for the study, and of those, one did not complete a baseline study questionnaire. All 69 patients who completed a baseline questionnaire were male with a mean age of 59 (range $=24-83$ ) years, and $35.3 \%(24 / 68)$ were married or had a live-in partner. In considering the potential applicability of the telemedicine system to home care, the majority of patients $(97.1 \%$, $67 / 69)$ lived at home rather than in a nursing home. Their living situation varied, with $41.3 \%$ (26/63) living without assistance, while the majority $(58.7 \%)$ received some kind of assistance or care at home $(39.7 \%$ had a full- or part-time caregiver, $12.7 \%$ had some assistance, and $6.3 \%$ used a full-time nurse). In addition, the majority $(63.3 \%)$ of participating patients considered their overall health to be "good or very good," $23.3 \%$ as "fair," and $13.3 \%$ "poor." There were no significant differences between the two participating sites in the demographic composition of the sample.

\section{Patient attitudes}

At visit 1, 69 of 70 patients completed the patient satisfaction questionnaire, and 44 of 48 patients at visit 3 . Table 1 shows the distribution of patient responses to each of the seven items in the questionnaire. Overall, both pre- and post-exposure to the telemedicine system, patients were generally confident in the telemedicine evaluation, and the majority considered the quality of care to be the same or better than seeing a physician in person. In particular, at both visits, although the majority (more than $85 \%$ ) thought it was important to be seen in person by a doctor for a pressure sore, less than $12 \%$ were not confident that the doctor would "know about their wound" using the telemedicine system instead of seeing the wound in person. In terms of quality of care, $78 \%$ at visit 1 and $76 \%$ at visit 3 felt that using telemedicine at home would be "the same or better" than visiting their physician. An even greater percentage $(98 \%$ at visit 1 and $89 \%$ at visit 3$)$ felt that telemedicine at home would be the same or better than visiting their physician in terms of respecting privacy. To the statement of "pictures and measurements the nurse took made me feel more self-conscious than when the physician looks at and touches my sore," more than $80 \%$ of the patients had no opinion or disagreed with the statement at each visit. Using telemedicine at home was considered the same or better than staying in the hospital and the same or better than recovering in an extended care facility by more than $80 \%$ at both visits.

\section{Change in patient attitudes}

Before evaluating the change in patient attitudes before and after exposure to the telemedicine system, we compared the demographic and clinical characteristics of those who completed the attitudinal questionnaires at both visits with those who completed the questionnaires during the first visit only. We found no statistically significant differences in marital status, nursing home residency, living situation, or overall health. Of note, patients who completed questionnaires at both visits tended to be older; $82 \%$ of the 44 who completed the survey at both visits were 50 years or older, compared to $56 \%$ of the 25 who completed the questionnaire during the first visit only ( $p=$ $0.104)$. The distribution of wound type was significantly different (Table 2; $p=0.002$ ) between those who completed both visits versus first visit only. Patients who completed the questionnaire at both visits tended to have more serious wound types (stages 3 and 4 pressure ulcers) than the patients who completed it at the first visit only: $65.9 \%(29 / 44)$ versus $40.0 \%(10 / 44)$. Therefore, those who completed the questionnaire at both visits tended to have more serious wounds as compared to their counterparts.

When using all data from both visits (as shown in Table 1) and ignoring the paired na- 
Table 1. Distribution of Patient Responses to Questions on Attitudes Toward Telemedicine

\begin{tabular}{|c|c|c|}
\hline & \multicolumn{2}{|c|}{ Number (\%) } \\
\hline & Visit 1 & Visit 3 \\
\hline \multicolumn{3}{|c|}{$\begin{array}{l}\text { 1. The pictures and measurements the nurse took made me feel more self- } \\
\text { conscious than when the physician looks at and touches my sore: }\end{array}$} \\
\hline Strongly disagree & $20(29.4)$ & $12(27.3)$ \\
\hline Disagree & $20(29.4)$ & $24(54.5)$ \\
\hline No opinion & $15(22.1)$ & $4(9.1)$ \\
\hline Agree & $12(17.6)$ & $3(6.8)$ \\
\hline Strongly agree & $1(1.5)$ & $1(2.3)$ \\
\hline Total & 68 & 44 \\
\hline \multicolumn{3}{|c|}{$\begin{array}{l}\text { 2. Using telemedicine at home would be worse, same, or better than } \\
\text { staying in the hospital: }\end{array}$} \\
\hline Worse & $8(13.6)$ & $6(14.0)$ \\
\hline The same & $14(23.7)$ & $17(39.5)$ \\
\hline Better & $37(62.7)$ & $20(46.5)$ \\
\hline Total & 59 & 43 \\
\hline \multicolumn{3}{|c|}{$\begin{array}{l}\text { 3. Using telemedicine at home would be worse, same, or better than } \\
\text { recovering in an extended care facility: }\end{array}$} \\
\hline Worse & $8(13.3)$ & $8(19.5)$ \\
\hline The same & $15(25.0)$ & 8 (19.5) \\
\hline Better & $37(61.7)$ & $25(61.0)$ \\
\hline Total & 60 & 41 \\
\hline \multicolumn{3}{|c|}{$\begin{array}{l}\text { 4. Using telemedicine at home would be worse, same, or better than } \\
\text { visiting your physician in terms of the quality of care: }\end{array}$} \\
\hline Worse & $13(22.4)$ & $10(23.8)$ \\
\hline The same & $34(58.6)$ & $24(57.1)$ \\
\hline Better & $11(19.0)$ & $8(19.0)$ \\
\hline Total & 58 & 42 \\
\hline \multicolumn{3}{|c|}{$\begin{array}{l}\text { 5. Using telemedicine at home would be worse, same, or better than } \\
\text { visiting your physician in terms of respecting privacy: }\end{array}$} \\
\hline Worse & $1(1.7)$ & $5(11.4)$ \\
\hline The same & $48(81.4)$ & $27(61.4)$ \\
\hline Better & $10(16.9)$ & $12(27.3)$ \\
\hline Total & 59 & 44 \\
\hline \multicolumn{3}{|c|}{$\begin{array}{l}\text { 6. How important is it to you to be seen, in person, by a doctor for a } \\
\text { pressure sore? }\end{array}$} \\
\hline Not important & $8(13.3)$ & $6(13.6)$ \\
\hline Important & $16(26.7)$ & $18(40.9)$ \\
\hline Very important & $36(60.0)$ & $20(45.5)$ \\
\hline Total & 60 & 44 \\
\hline \multicolumn{3}{|c|}{$\begin{array}{l}\text { 7. How confident are you that the doctor will be able to know about your } \\
\text { pressure sore by the use of this equipment instead of seeing it in person? }\end{array}$} \\
\hline Very unconfident & $0(0)$ & $1(2.3)$ \\
\hline Somewhat unconfident & $6(9.2)$ & $4(9.3)$ \\
\hline No opinion & $18(27.7)$ & $6(14.0)$ \\
\hline Somerwhat confident & $23(35.4)$ & $20(46.5)$ \\
\hline Very confident & $18(27.7)$ & $12(27.9)$ \\
\hline Total & 65 & 43 \\
\hline
\end{tabular}

Italicized responses are responses considered to be associated with positive attitudes for telemedicine.

ture of the data, patient attitudes were similar between time periods, except for the questions on feelings of self-consciousness (question 1) and privacy (question 5). The percentage of patients who disagreed or strongly disagreed with the statement of feeling self-conscious increased from $59 \%$ to $82 \%(p=0.038)$, suggest- ing that patients became more comfortable with telemedicine after using the system. Regarding privacy, the percentage of patients who responded that telemedicine is "the same" as seeing a physician in person decreased from $81 \%$ to $61 \%$, while the percentage of both "worse" and "better" responses increased $(p=0.035)$, 
TAble 2. Distribution of Wound Type For Patients Who Completed Questionnaires at Both Visits and First Visit Only

\begin{tabular}{lcc}
\hline & \multicolumn{2}{c}{ Number (\%) of patients } \\
\cline { 2 - 3 } Wound type & Both visits & First visit only \\
\hline Vascular & $5(11.4)$ & $11(44.0)$ \\
Postoperative & $7(15.9)$ & $0(0.0)$ \\
Stage 2 pressure & $3(6.8)$ & $4(16.0)$ \\
Stage 3/4 pressure & $29(65.9)$ & $10(40.0)$ \\
$\quad$ Total & $44(100.0)$ & $25(100.0)$ \\
\hline
\end{tabular}

Wound type was based on the nature of the wound at visit 1 .

suggesting that more patients formed opinions, both positive and negative, regarding privacy after exposures to telemedicine. At visit 3, however, in terms of respecting privacy, still a larger percentage of patients viewed telemedicine to be better $(27 \%)$, rather than worse $(11 \%)$, than visiting a physician in person.

Shown in Table 3 are results of alternative analyses comparing attitudes at the two different time periods using data only of patients who completed questionnaires at both visits. Patient attitudes generally agreed between time periods. Change-scores between visits were not statistically significant, with the median change-score of 0 for each of the seven questions. The mostly positive mean changescores, however, suggest that any changes observed were generally in the direction of improved attitude. When looking at those who changed their perception after exposure to the telemedicine system, for five of the seven questions, more patients showed improvement rather than worsening. In particular, for the perceptions of quality of care (question 4), privacy (question 5), and confidence (question 7), the numbers of people who improved were two times or greater compared with the number of people who worsened. In fact, patient attitude regarding privacy significantly improved after exposure ( $p=0.02$; test of symmetry). On the other hand, a greater percentage of patients' attitudes worsened rather than improved toward using telemedicine at home instead of staying in the hospital (question 2), and toward using telemedicine at home instead of recovering in an extended care facility (question 3).

\section{Telemedicine attitude scale}

To determine whether a telemedicine attitudinal scale could be developed from the items in the questionnaire, we calculated Cronbach's $\alpha$ and conducted a correlation analysis using all seven questions in the questionnaire. Initially, scores given to the responses to each question ranged from 1 to 3 (questions 2-6) or 1 to 5 (questions 1 and 7) (see Table 1). Questions 1 and 6 were scored in reverse order so that a larger score could be consistently interpreted as favorable attitudes toward telemedicine. The first question, concerning feelings of self-consciousness, was dropped because it correlated poorly with the others $(r=0.07$ at visit 1 and 0.21 at

Table 3. Analyses of Change in Attitudes Between Visit 1 and Visit 3

\begin{tabular}{|c|c|c|c|c|c|c|c|}
\hline \multirow[b]{2}{*}{ Question $^{\mathrm{a}}$} & \multirow[b]{2}{*}{ Median } & \multirow[b]{2}{*}{$I Q R^{\mathrm{b}}$} & \multirow[b]{2}{*}{ Mean } & \multicolumn{2}{|c|}{ Number (\%) } & \multirow[b]{2}{*}{ Ratio $^{\mathrm{c}}$} & \multirow[b]{2}{*}{$\mathrm{P}$ value } \\
\hline & & & & Improved & Worsened & & \\
\hline 1. Self-consciousness & 0 & 1 & 0.16 & $27.9(12 / 43)$ & $20.9(9 / 43)$ & 1.33 & 0.43 \\
\hline 2. Hospital & 0 & 1 & -0.08 & $19.4(7 / 36)$ & $33.3(12 / 36)$ & 0.58 & 0.18 \\
\hline 3. Extended care & 0 & 0 & 0 & $14.3(5 / 35)$ & $20.0(7 / 35)$ & 0.71 & 0.34 \\
\hline 4. Quality of care & 0 & 0 & 0.17 & $20.0(7 / 35)$ & $5.7(2 / 35)$ & 3.50 & 0.48 \\
\hline 5. Privacy & 0 & 0 & 0.14 & $21.6(8 / 37)$ & $10.8(4 / 37)$ & 2.00 & 0.02 \\
\hline 6. Importance & 0 & 1 & 0.15 & $29.0(11 / 38)$ & $15.8(6 / 38)$ & 1.83 & 0.13 \\
\hline 7. Confidence & 0 & 1 & 0.21 & $29.3(12 / 41)$ & $12.2(5 / 41)$ & 2.40 & 0.10 \\
\hline
\end{tabular}

The change score was calculated so that a positive value reflects an improvement in attitudes toward telemedicine.

a Refer to Table 1 for a full description of each attitudinal question.

bInterquartile range (75th percentile-25th percentile).

'Ratios are calculated as percent improved divided by percent worsened (or number improved divided by number worsened).

$\mathrm{d}_{p}$ values from test of symmetry; $p<0.05$ indicates for asymmetry between periods. 
visit 3). Finally, we modified the scoring scheme to create proper summary scales. The five responses were collapsed to three.

Table 4 shows the inter-question reliability results using the described scoring method. For visit 1 data, all six questions appear to fit well, with an $\alpha$ coefficient of 0.69 for the six-question summary scale. For visit 3 data, however, the $\alpha$ coefficient was lower at 0.53 . In particular, questions 6 and 7 did not fit well, as evidenced by a low correlation with the rest of the questions. When questions 6 and 7 were both removed, the $\alpha$ coefficient was 0.67 for visit 1 data, but increased substantially from 0.53 to 0.69 for visit 3 data, again indicating lack of fit for questions 6 and 7 for visit 3 .

Based on these findings, two summary scores were developed as averages of the item scores: one using six questions (questions 2-7) and another using four questions (questions 2-5). The summary scores range from 1 to 3 , with a score close to 3 corresponding to a positive attitude, 2 indicating neutrality, and close to 1 corresponding to a negative attitude. Several analyses were conducted with these summary scores. A paired $t$ test comparing the attitude summary score differences between visits 1 and 3 showed almost no change using summary scores based on either six questions or four questions, with an average decrease of $0.03(p=0.63)$ for the former and an increase of $0.05(p=0.50)$ for the latter. The summary scores, therefore, did not show any change in overall attitude after exposure to data collection.

Table 5 shows the comparison of the fourquestion summary scores across the four differ- ent wound types at visits 1 and 3 . At both visits and for all wound types, the attitudes were more positive than neutral. In particular, when the mean summary scores were compared with a hypothesized value of 2 (a value indicating neutral attitude) using a one-sample $t$ test, the summary scores showed that at both visit 1 and 3 , the overall attitude scores of $2.26(p<0.01)$ and $2.22(p<0.01)$, respectively, were better than neutral. None of the comparisons across wound types or between visits was statistically significant. However, the scores for patients with vascular wounds tended to be higher than those with other wound types for both visits.

\section{Time spent}

The time the nurse coordinators spent in collecting data was significantly longer during the first visit than during subsequent visits. Table 6 presents the median nursing time spent by wound type and visit order. Median, instead of mean, is shown, because the distribution of time spent was slightly right-skewed. To evaluate the trend in time spent over visits, it was necessary to adjust the analysis for wound type, because wound type appeared to be associated with both time spent and visit number. A random-effects model was fit to model the visit effect on nursing time while adjusting for within-person correlation, with time spent as the dependent variable, an indicator for first visit, three indicators for four wound types, and visit number as the independent variables. According to the model (not shown), after adjusting for wound type, the decrease in time

Table 4. Cronbach's Coefficient $\alpha$ with Deleted Questions, Visits 1 and 3, with Question 1 Removed

\begin{tabular}{lcccc}
\hline & \multicolumn{2}{c}{ Visit 1} & & Visit 3 \\
\cline { 2 - 5 } $\begin{array}{l}\text { Deleted } \\
\text { question }\end{array}$ & $\begin{array}{c}\text { Correlation } \\
\text { with rest }\end{array}$ & $\begin{array}{c}\text { Cronbach's } \\
\alpha\end{array}$ & $\begin{array}{c}\text { Correlation } \\
\text { with rest }\end{array}$ & $\begin{array}{c}\text { Cronbach's } \\
\alpha\end{array}$ \\
\hline 2 & 0.41 & 0.64 & 0.42 & 0.40 \\
3 & 0.60 & 0.60 & 0.41 & 0.46 \\
4 & 0.45 & 0.64 & 0.41 & 0.42 \\
5 & 0.53 & 0.63 & 0.10 & 0.56 \\
6 & 0.26 & 0.70 & 0.03 & 0.59 \\
7 & 0.39 & 0.65 & & 0.53 \\
\hline
\end{tabular}

For visit 1, responses received for each question ranged from 58 to 65 patients. For visit 3 , responses received ranged from 41 to 44 patients.

aRefer to Table 1 for a full description of each attitudinal question. 
Table 5. Attitude Summary Score Based on Four Questions, by Visit and Wound Type

\begin{tabular}{lccrr}
\hline $\begin{array}{l}\text { Wound type at } \\
\text { visit 1 }\end{array}$ & Visit 1 & Visit 3 & $\begin{array}{c}\text { Change } \\
\text { (visit 3-visit 1) }\end{array}$ & $\begin{array}{c}\mathrm{p} \text { value } \\
(\text { change }=0)^{\mathrm{a}}\end{array}$ \\
\hline Vascular & $2.44(13,0.38)$ & $2.40(5,0.22)$ & $0(5,0.35)$ & 1.00 \\
Postoperative & $2.11(7,0.43)$ & $2.17(13,0.57)$ & $-0.11(7,0.43)$ & 0.55 \\
Stage 2 pressure & $2.28(8,0.41)$ & $2.18(7,0.24)$ & $-0.33(3,0.63)$ & 0.41 \\
Stage 3/4 pressure & $2.21(32,0.53)$ & $2.22(19,0.59)$ & $0.16(23,0.45)$ & 0.14 \\
$\quad$ All types & $2.26(60,0.48)$ & $2.22(44,0.50)$ & $0.05(38,0.46)$ & 0.54 \\
$\quad$ P value & 0.16 & 0.26 & 0.74 & \\
\hline
\end{tabular}

Data are mean ( $n, \mathrm{SD})$ values. The four questions summarized are 2, 3, 4, and 5 (see Table 1 for details).

${ }^{\text {a The }} p$ values are from testing for no change in summary scores across visit for each wound type using the Wilcoxon signed rank test for paired data.

bSeven of the 23 patients in this category were postoperative by visit 3 . The mean change in summary score was $0.14(p=0.31)$ without the seven patients.

cThe $p$ values are from testing for mean difference across wound type at each visit using nonparametric median tests.

spent was significant after the first visit (mean difference $=25.2 \mathrm{~min}, p<0.01$ ), but not beyond, and stage $3 / 4$ pressure ulcers took significantly longer time (mean difference $=19.7$ min, $p<0.01$ ) than post-operative or stage 2 pressure ulcers.

Data entry/upload times averaged 98.5 $(\mathrm{SD}=637.2) \mathrm{min}$. For $61.3 \%$ of the visits, less than 30 min was spent on data entry and uploading. Finally, the assessments conducted by the telemedicine physicians averaged 2.6 (S.D. = 5.1) $\mathrm{min}$.

\section{DISCUSSION}

This study revealed that patients were generally comfortable with the use of a telemedicine system to assess wound status. Specifically, less than $25 \%$ of the patients felt that using telemedicine at home would be "worse" than visiting their physician in-person in terms of the quality of care, even though about $85 \%$ of the patients felt that it was important or very important to be seen in person by a doctor for a pressure sore. Perhaps more importantly, less than $12 \%$ of the patients felt "somewhat" or "very unconfident" that their doctors would be able to know about the pressure sore by the use of this system instead of seeing the wound in person. In addition, using a satisfaction summary score, patients' overall attitude toward the telemedicine system was more positive than neutral. These findings suggest that the ideal system of care, from a patient perspective would be routine monitoring using the telemedicine system with occasional in-person visits to a physician.

The patients sampled in this study were assessed in-person by a physician and, at the same time, were exposed to telemedicine. Thus, this study's assessment of attitudes toward telemedicine is based on patients who had immediate access to an in-person physician evaluation, rather than on patients from remote areas, who might have difficulty traveling to see

Table 6. Median (25th Percentile, 75th Percentile) for Nursing Time Spent with Patient at First Visit and at Subsequent Visits

\begin{tabular}{llcrr}
\hline Wound type & $\begin{array}{c}\text { Visit } 1 \\
(\mathrm{n}=54)\end{array}$ & $\begin{array}{c}\text { Visit 2 } \\
(\mathrm{n}=49)\end{array}$ & $\begin{array}{c}\text { Visit 3 } \\
(\mathrm{n}=41)\end{array}$ & $\begin{array}{c}\text { Visits 4, 5, and 6 } \\
(\mathrm{n}=86)\end{array}$ \\
\hline Vascular & $60(47.5,83)$ & $34(30,42.5)$ & $40(40,40)$ & $35(30,75)$ \\
Postoperative & $60(55,60)$ & $35(30,45)$ & $32.5(30,50)$ & $30(30,30)$ \\
Stage 2 pressure & $70(15,120)$ & $40(30,45)$ & $42.5(30,45)$ & $30(26,45)$ \\
Stage 3/4 pressure & $85(60,105)$ & $47.5(37.5,75)$ & $50(40,60)$ & $50(35,90)$ \\
$\quad$ Total & $75(60,95)$ & $40(30,60)$ & $40(30,55)$ & $30(30,58)$ \\
\hline
\end{tabular}

Data are in minutes. 
a physician. It is possible that attitudes toward telemedicine would be even more positive for patients in remote areas. Although conducted more than 5 years ago, in a study ${ }^{6}$ of rural West Virginia adults, however, "nearly two-thirds thought patients would find telemedicine in general to be less satisfactory than seeing a physician in person."

After some exposure to the telemedicine data collection, patients tended to be more positive about the quality of care and privacy using telemedicine at home, and were more confident about the doctor's knowledge about their wound when using the telemedicine system. Hence, some preliminary education about telemedicine may help ensure that patients are both comfortable and satisfied with this method of health care delivery. However, such exposure did not improve attitudes toward using telemedicine at home instead of staying in an extended care facility or hospital.

Our findings are consistent with other published work on patient satisfaction with storeand-forward telemedicine applications. While generally positive about these applications, patients have some concerns with the lack of direct communication with a physician. However, considerably more research needs to be done in this area, given the dearth of systematic studies of patient satisfaction with this telemedicine modality.

The results also indicate that considerable effort is required for collecting and transmitting the telemedicine data. At the same time, a significant decrease was seen in time spent collecting the telemedicine data from first visit to subsequent visits. As the use of digital images in computer-based medical records increases, along with inter-facility communication of medical record data, this time requirement should decrease significantly.

\section{ACKNOWLEDGMENTS}

This work was supported by a grant from the Department of Veterans Affairs Health Ser- vices Research and Development Service (Project Number ACC 97-013-3). We are grateful to the physicians who participated in the study: Kenna Given, Jay Lucas, Kit Outlaw, Riley S. Rees, Mary D. Thomas, and Rose Trincher. We would also like to extend our appreciation to the nurse coordinators: Ellen Clements, Mary Connors, and Cindy Hoganson.

\section{REFERENCES}

1. Smith DM, Winsemius DK, Besdine RW. Pressure sores in the elderly: can this outcome be improved? IGen Intern Med 1991;6:81-93.

2. Siegler EL, Lavizzo-Mourey R. Management of stage III pressure ulcers in mdoerately demented nursing home residents. L Gen Intern Med 1991;6:507-513.

3. Agency for Health Care Policy and Research. Clinical practice guideline number 15: treatment of pressure ulcers. AHCPR Publication 95-0652. Rockville, MD: U.S. Department of Health and Human Services, 1994.

4. Kim HM, Lowery JC, Hamill JB, Wilkins EG. Accuracy of Web-based system for monitoring chronic wounds. Telemed J e-Health 2003;9:129-140.

5. Allen A, Ermer D, Doolittle G, Whitten P, Zaylor C. Telemedicine in Kansas—beyond anecdotes [abstract]. Telemed J 1997;3:86.

6. Brick JE, Bashshur RL, Brick JF, D'Allessandri RM. Public knowledge, perception, and expressed choice of telemedicine in rural Virginia. Telemed I 1997;3:159171.

7. Cleves MA. sg74: symmetry and marginal homogeneity test/TDT. Stata Tech Bull 1997;40:23-27. [Stata Techn Bull Reprints 1998;7:193-197.]

8. Streiner DL, Norman GR. Health measurement scales: a practical guide to their development and use, 2nd ed. New York: Oxford University Press, 1995.

9. Laird NM, Ware JH. Random-effects models for longitudinal data. Biometrics 1982;38:963-974.

Address reprint requests to: H. Myra Kim, Sc.D. Center for Statistical Consultation and Research University of Michigan 3550 Rackham 915 East Washington Street Ann Arbor, MI 48109-1070

E-mail: myrakim@umich.edu 